\title{
Factors Affecting Adherence to Early Mobilization in an Intensive Care Unit: An Analysis of Data Collected Prospectively Over a Three-Year Period by Clinical Information System
}

\section{Paula Perelló}

Joan XXIII University Hospital in Tarragona: Hospital Universitari de Tarragona Joan XXIII Josep Gómez

Joan XXIII University Hospital in Tarragona: Hospital Universitari de Tarragona Joan XXIII Judith Mariné

Joan XXIII University Hospital in Tarragona: Hospital Universitari de Tarragona Joan XXIII Maria Teresa Cabas

Joan XXIII University Hospital in Tarragona: Hospital Universitari de Tarragona Joan XXIII

Alba Arasa

Joan XXIII University Hospital in Tarragona: Hospital Universitari de Tarragona Joan XXIII

\section{Zaida Ramos}

Joan XXIII University Hospital in Tarragona: Hospital Universitari de Tarragona Joan XXIII

\section{David Moya}

Joan XXIII University Hospital in Tarragona: Hospital Universitari de Tarragona Joan XXIII

\section{Irene Reynals}

Joan XXIII University Hospital in Tarragona: Hospital Universitari de Tarragona Joan XXIII

\section{María Bodí}

Joan XXIII University Hospital in Tarragona: Hospital Universitari de Tarragona Joan XXIII

\section{Mònica Magret ( $\square$ mmagret@gmail.com )}

Joan XXIII University Hospital in Tarragona: Hospital Universitari de Tarragona Joan XXIII https://orcid.org/0000-0002-9534-9920

\section{Research}

Keywords: early mobilization, clinical information systems, critical illness, safety, intensive care unit, adherence to protocols.

Posted Date: October 29th, 2021

DOl: https://doi.org/10.21203/rs.3.rs-983842/v1 
License: (c) (i) This work is licensed under a Creative Commons Attribution 4.0 International License. Read Full License 
Factors affecting adherence to early mobilization in an intensive care unit: an analysis of data collected prospectively over a three-year period by clinical information system

Paula Perelló1*, Josep Gómez ${ }^{2 *}$, Judith Mariné1, Maria Teresa Cabas ${ }^{1}$, Alba Arasa $^{1}$, Zaida Ramos ${ }^{1}$, David Moya ${ }^{3}$, Irene Reynals ${ }^{3}$, María Bodí ${ }^{4}$, Mònica Magret $^{5}$

*These authors contributed equally to this work.

Affiliations:

${ }^{1}$ Intensive Care Unit, Hospital Universitari Joan XXIII/IISPV, Tarragona, Spain

${ }^{2}$ Intensive Care Unit, Hospital Universitari Joan XXIII/IISPV/URV, Tarragona, Spain

${ }^{3}$ Rehabilitation, Hospital Universitari Joan XXIII, Tarragona, Spain

${ }^{4}$ Intensive Care Unit, Hospital Universitari Joan XXIII/IISPV/URV, CIBERes, Tarragona, Spain

5 Intensive Care Unit, Hospital Universitari Joan XXIII/IISPV/URV, Tarragona, Spain. Corresponding author: mmagret@gmail.com 


\section{$\underline{\text { ABSTRACT }}$}

Background: Early mobilization benefits critically ill patients. Scant information is available about adherence to early mobilization protocols in intensive care units (ICU). With the aim of optimizing the application of our early mobilization protocol in mechanically ventilated patients in routine daily practice, this study analyzed adherence to the protocol, impediments to adherence, and adverse events in our ICU.

Methods: This observational study analyzed data collected prospectively at a 24-bed polyvalent ICU over a three-year period (2017-2019). Data from adult patients on mechanical ventilation $>48$ hours who met the inclusion criteria for the early mobilization protocol were included. We analyzed demographics, adherence to the protocol, total number of mobilizations, impediments to early mobilization, artificial airway/ventilatory support at each mobilization level, and adverse events during mobilization. All data was automatically obtained from the clinical information system by extract, transform, and load processes using Python 3.0. The unit of analysis was ICU stay-day.

Results: We analyzed 3269 stay-days from 388 patients with median age of 63 (51-72) years, median APACHE II 23 (18-29) and median ICU stay of 10.1 (6.2-16.5) days. Adherence to the protocol was 56.6\% (1850 stay-days), but patients were mobilized in only $32.2 \%$ (1472) of all stay-days. The most 
common reasons for not mobilizing patients were failure to meeting the criteria for clinical stability in $241(42 \%)$ stay-days and unavailability of physiotherapists in 190 (33\%) stay-days. Adverse events occurred in only 6 $(0.4 \%)$ stay-days mobilizations.

Conclusions: Although adherence to the protocol was high, patients were mobilized in only one-third of all stay-days. This study points to ways we can improve early mobilization in our ICU, including assessing the suitability of the criteria for clinical stability and increasing the presence of physiotherapists.

Keywords: early mobilization, clinical information systems, critical illness, safety, intensive care unit, adherence to protocols. 


\section{Background}

Forced rest in intensive care units (ICU) often results in muscle weakness within days. The term ICU-acquired weakness refers to myopathy and/or polyneuropathy secondary to critical illness and immobilization among other factors[1,2]. ICU-acquired weakness occurs in $25 \%$ to $50 \%$ of ventilated patients [3]. Bed rest is also associated with long-term complications, manifesting as post-intensive care syndrome comprising physical, cognitive, and psychiatric sequelae that prevent patients from recovering their prior quality of life after discharge from the ICU[4].

Although the complications of bed rest and the benefits of mobilization have been known for decades, early mobilization (EM) was first implemented in ICUs only 20 years ago[5,6]. EM is safe and feasible[7,8], and it is associated with better outcomes, including improved functional status, shorter duration of mechanical ventilation (MV), lower incidence of delirium, increased muscle strength, and better quality of life[9,10].

Advances in technology and medicine have increased the complexity of critical care medicine, making clinical information systems (CIS) useful to ensure safe, timely, and effective care. CIS include databases, allowing us to measure what 
we do, analyze adherence to recommendations based on scientific evidence, improve professional performance, and evaluate the impact of improvement strategies[11].

Although some studies have evaluated the practice of EM in ICU[12,13], few have evaluated daily mobilization practices in mechanically ventilated patients[12], and most of these measured adherence at a single point in time[13]. To our knowledge, no reports of longitudinal studies evaluating mobilization practices in MV patients in routine clinical practice have been published. The current study aimed to analyze EM in our ICU over a 3-year period. To this end, we used data from our CIS database to determine adherence to EM protocols, the incidence of adverse events, the role of ventilator support, and impediments to mobilization.

\section{Methods}

\section{Patients and setting}

This observational study analyzed data collected prospectively at a 24-bed polyvalent ICU at a tertiary university hospital during a three-year period (January 2017-December 2019). We included data from patients aged $\geq 18$ years with Barthel Index $(\mathrm{BI})>70$ prior to admission to the ICU who required 
$>48$ hours mechanical ventilation. We excluded data from patients with spinal cord injuries, history of neuromuscular disease, body mass index $(\mathrm{BMI})>45$, unsafe airways, cardiac arrests, traumatic brain injuries, or strokes. We discarded stay-days that occurred after decisions to limit life support.

All patients or their legal representatives provided written informed consent, and our center's research ethics committee approved the study protocol[14].

\section{Endpoints and definitions}

The unit of analysis was ICU stay-day. The primary endpoint was adherence to the EM protocol, defined as the percentage of stay-days in which the EM form extracted from the CIS contained information regarding the type of mobilization or justified reasons for not mobilizing the patient. Secondary endpoints were (a) total number mobilizations, defined as the percentage of stay-days in which patients were mobilized in relation to total stay-days; (b) putative hidden adherence, defined as the percentage of stay-days in which the mobilization form had not been completed, but patients did not meet clinical stability criteria for mobilization; (c) delay in beginning the EM protocol, defined as stay-days before begining the EM protocol in which the EM form was not completed and patients met de clinical stability criteria; (d) impediments to mobilization; (e) levels of mobilization according to the ventilatory support, type of airway and 
level of sedation; (f) analysis of non-working days, and (g) adverse events during mobilization, defined as arrhythmias, falls, accidental removal of devices, hypertension (systolic blood pressure> $200 \mathrm{mmHg}$ ), hypotension (systolic blood pressure $<80 \mathrm{mmHg}$ ), hypoxemia $(\mathrm{SaO} 2<80 \%)$, and accidental extubation [7].

\section{Protocol workflow}

A multidisciplinary team comprising intensivists, physiotherapists, rehabilitators, and nurses screened all candidates for EM daily (except on weekends and holidays) and began to implement the EM protocol as soon as the patient met the criteria for clinical stability, which were adapted from expert consensus and recommendations [15]. The protocol comprised five levels: Level 1 was limited to passive mobilizations of the limbs, postural changes, and sitting up in bed; Level 2 included active-assisted mobilizations of the limbs; Level 3 focused on trunk control and included sitting on the edge of bed and passive transfer to the chair; Level 4 involved active transfer to the chair and standing; and Level 5, ambulation (Annex 1). Physiotherapists's assessments determined the initial level of EM for each patient and their readiness to progress to the next level. 


\section{Data collection and representation}

All variables were extracted from data routinely stored in our CIS (Centricity Critical Care ${ }^{\circledR}$, GE Healthcare; Chicago, IL, USA) through automatic capture from connected devices, automatic entry from integrated information systems [laboratory results and data from our hospital's electronic health record ( $\mathrm{SAP}^{\circledR}$; Walldorf, Germany)], and manual entry (Annex 2). Depending on their type and source, data are stored in different tables in the CIS database. Each table contains at least one key field that enables the information it contains to be connected with information in other tables; this relational schema makes it possible to integrate information from different data types and sources with extract, transform, and load (ETL) processes. We used Python 3.0 to execute the ETL process that generated our final dataset from the raw tables within our CIS database (Fig. 1).

The ETL process allowed us to automatically obtain a large dataset in which each row contains all the relevant data for each patient stay, including both static (patient-related) and dynamic (stay-related) data. Static data included age, sex, comorbidities, Acute Physiological and Chronic Health Evaluation (APACHE) II score at admission, Sequential Organ Failure Assessment (SOFA) score at admission, reason for admission, ICU length of stay (LOS), and hospital LOS. 
Dynamic data included data from the EM form stored in the CIS (Figure 1), artificial-airway/ventilatory-support data, and all the data required to determine whether patients met the criteria for clinical stability on each day of the stay. To facilitate daily decisions related to the EM protocol, the ETL automatically determined whether these criteria were met based on the data recorded between 8 am and 10 am.

\section{Sedation}

To analyze patients' level of sedation during EM, we used the Richmond Agitation-Sedation Scale (RASS).

\section{Statistical analysis}

To determine adherence to the protocol and impediments to EM, we performed a descriptive analysis of the data. Numerical variables are expressed as median and interquartile range, and categorical variables are expressed as frequencies and percentages. All data mining and processing were carried out using Python 3.0 . 


\section{Results}

Of the 2924 patients admitted to the ICU during the study period, $1231(42,1 \%)$ required mechanical ventilation $(\mathrm{MV})$; of these $771(62,6 \%)$ received $\mathrm{MV}>48$ hours and after excluded $383(49,7 \%)$ for meeting the exclusion criteria, we included 388 (50,3\%) patients (Fig. 2) (median age, 63 [51-72] y; $273(70.4 \%)$ men). At admission, the median APACHE II score was 23 (18-29) and the median SOFA score was $3(1-5.8)$. The most frequent reason for admission was respiratory failure $23.5 \%$, followed by postsurgical care $19.8 \%$ and septic shock $18.3 \%$.

\section{Primary outcome}

The ICU stays for these 388 patients comprised a total of 4568 stay-days; of these, 1299 fell on weekends or holidays when patients were not screened for EM. Thus, a total of 3269 stay-days were analyzed to obtain the adherence (Fig. 3). The EM form was completed in 2040 stay-days; of these, patients were mobilized in 1472 stay-days. Since the lack of a physiotherapist in 190 staydays is a cause of non-adherence to the protocol, they were not taken into account within the 2040 stay-days, so the adherence was 1850 stay-days (56.6\%) compared to the 3269 stay-days evaluated. 


\section{Secondary outcomes}

\section{Total number of mobilizations}

When all 4568 stay-days were considered to calculate the total number of mobilizations patients underwent EM in only 1472 (32.2\%) stay-days.

\section{Putative hidden adherence}

The EM form was not completed in 1229 stay-days (Fig. 3). In 509 of these stay-days, patients did not meet the criteria for clinical stability and would have been ineligible for EM. Since failure to complete the form in these cases was certainly an oversight because clinicians were totally aware of patients' ineligibility, generating hidden protocol adherence of $15.6 \%$, which would increase adherence to $72.2 \%$.

\section{Delay in beginning}

In the remaining 720 stay-days, patients meet the criteria for clinical stability and of these 285 stays-days were days of delay in the beginning of the protocol. The median ICU length of stay in the patients who underwent EM was 10.1 (6.2-16.5) days. EM was initiated a median of 3 (1-5) days after admission to the ICU, and active mobilization (level II) was initiated a median of 6 (4-10) days after admission. 


\section{Impediments to mobilization}

To obtain the impediments to mobilization we analyzed 568 stay-days in which the patients were not mobilized (Fig. 3). The reasons were: failure to meet the criteria for clinical stability in $241(42,2 \%)$ stay-days, unavailability of physiotherapists in 190 (33,3\%) stay-days, patient's behavior in 88 (17\%) staydays, unknown - i.e., the form was completed but mobilization did not take place and no reason was recorded in $43(8 \%)$ stay-days. Failure to meet the criteria for clinical stability was due to hemodynamic factors in $121(50.2 \%)$ stay-days, respiratory factors in 81 (33.6\%) stay-days, neurologic factors in 27 $(11.2 \%)$ stay-days, and infectious factors in $12(5 \%)$ stay-days.

Levels of mobilization according to the ventilatory support, the type of airway and level of sedation

Finally, we analyzed the levels of mobilization according to the ventilatory support and the type of airway (Table 1). Mobilizations took place in bed (Level I and II) in 972 (66\%) stay-days and out of bed (Level III-V) in 500 (34\%) staydays. In mobilizations that took place in bed, patients were on invasive mechanical ventilation in 707 (48\%) stay-days (with an endotracheal tube in place in 467 (66\%) stay-days and with a tracheostomy tube in place in 240 
(34\%) stay-days), without ventilatory support in 179 (12.1\%) stay-days, on high-flow nasal cannula in 53 (3.6\%) stay-days, and noninvasive mechanical ventilation in $33(2.3 \%)$ stay-days.

In mobilizations that took place out of bed, patients were without ventilatory support in 327 (22.2\%) stay-days, on invasive mechanical ventilation in 105 (7.1\%) stay-days (with an endotracheal tube in place in $31(30 \%)$ stay-days and a tracheostomy tube in place in 74 (70\%) stay-days), on high-flow nasal cannula in $40(2.7 \%)$ stay-days, and on noninvasive mechanical ventilation in $28(2 \%)$ stay-days. Of the 105 stays with artificial airway and connected to MV or mechanical ventilation-tracheostomy (MV-T), 50 stay-days sitting in bed (level 3), 17 stays sitting out of bed (level 4) and 39 stay-days standing out of bed and walking (level 5).

In 530 stay-days where Level I mobilizations took place, 439 (82.7\%) stay-days patients were sedated (RASS $\leq-1), 25(4.8 \%)$ stay-days were agitated (RASS $\geq 1$, and $66(12.5 \%)$ stay-days were awake and calm (RASS $=0)$.

\section{Analysis of non-working days}

In 1299 stay-days patients were not mobilized due to non-working days. Of these, in 851 (65.5\%) stay-days patients met de clinical stability criteria and in 
$448\left(34{ }^{\prime} 5 \%\right)$ stay-days patients failed to meet the clinical stability criteria.

\section{Advers events}

In the 1472 stay-days during the study period, $6(0.4 \%)$ adverse events occurred out-of-bed: 3 episodes of hypoxemia (quickly corrected with supplemental oxygen) and 1 each of orthostatic hypotension, hypertension, and arrhythmia. All adverse events were corrected by placing the patients in bed again. None of the adverse events resulted in complications that required other therapies.

\section{Discussion}

To determine impediments to the application of the EM protocol in our polyvalent ICU, this study used real-world data stored in the CIS to analyze factors related to adherence to the protocol during daily clinical practice over a three-year period. EM took place in $56.6 \%$ stay-days, showing that there is much room for improvement. The main impediment to EM was failure to meet the clinical stability criteria, followed by the unavailability of physiotherapists.

In a review of 40 studies, Dubb et al. [16] identified 28 impediments to EM, concluding that successful implementation of EM protocols requires each ICU to identify impediments based on its patient mix, equipment, staff expertise, and 
local culture. Most studies report that patient-related factors such as hemodynamic instability, delirium, or sedation levels are major impediments to EM. In our study, the main impediment to EM was failure to meet the safety criteria, which was the reason mobilization was not performed in half of the cases; these findings corroborate those reported in other studies $[7,12,17]$. Although consensus recommendations regarding the safety criteria for mobilizing mechanically ventilated patients can be used to guide rehabilitation in the ICU while minimizing the risk of adverse events [15], the absence of clinical practice guidelines suggests that cautious application of more flexible criteria guided by analyses of data from the CIS might enable more patients to benefit from EM.

EM in critically ill patients requires coordination, commitment, and physical effort by the multidisciplinary team. Physiotherapists play a key role in EM; however, our country's ICU culture assigns a limited role for physiotherapists compared to other countries[18]. A recent study in our country found that $18.6 \%$ of ICU teams included no physiotherapists; $10.5 \%$ had a physiotherapist for less than 5 hours a week, $12.8 \%$ had one between 5 and 10 hours a week, $10.5 \%$ between 10 and 15 hours a week, $8.1 \%$ between 15 and 20 hours a week, and only $4.6 \%$ had one for more than 24 hours a week. In the remaining $34.9 \%$, 
physiotherapists could be consulted through the rehabilitation service [19]. Our protocol was designed considering that physiotherapists would be present for 6 hours a day on weekdays that were not on non-working-days. Our results show that in 851 non-working stay-days patients met the safety criteria but were not mobilized because physiotherapists were unavailable.

One way to mitigate the unavailability of physiotherapists is to involve nurses and family members in EM. Integrating early mobility training into nursing education can help nurses understand the risks and benefits of mobilization in critically ill patients; increasing nurses' knowledge can improve outcomes through better management of personnel needed to complete transfers of patients being mobilized [20]. Rukstele et al. [21] found that the participation of family members in mobilization efforts increased adherence to recommendations for mobility in the ICU constantly over a 6-month period.

In our study, patients were still connected to invasive mechanical ventilation in $48 \%$ of mobilizations, and patients remained in bed in $66 \%$. This is logical, considering that patients were in the subacute phase of their disease and were sedated or being weaned from sedation. RASS scores show that patients were sedated in $82.7 \%$ of the stay-days in which Level I early mobilizations took place. Reviewing sedation protocols with the aim of shortening the time under 
sedation, as recommended in recent guidelines [22], might also improve the rate of early mobilizations. Nonetheless, $34 \%$ of mobilizations in our study were done outside the bed; of these, $22,2 \%$ took place after patients no longer required ventilatory support, although patients remained connected to mechanical ventilation in $7.1 \%$ of all mobilizations, mostly through tracheostomy tubes (5\% of all mobilizations). Recent point-prevalence studies have also found low rates of out-of-bed mobilizations. Although a German study reported that $24 \%$ of mobilizations took place outside bed, $50 \%$ of the patients in that study were on noninvasive mechanical ventilation. Another study, done in Australia and New Zealand, reported that none of the patients who required mechanical ventilation sat out of bed, stood, or walked, and only $12 \%$ sat over the side of the bed[5]. A recent study in Brazil reported that only 3 of 158 mechanically ventilated patients stood, marched on spot, or walked during mobilizations[13]. Although the rate of out-of-bed mobilizations in patients undergoing invasive mechanical ventilation in our study is low, it is to our knowledge the highest reported to date.

The lack of data in one-third of all stay-days reveals the need to establish mechanisms to audit routine data collection in our unit [23,24]. In the stay-days in which the EM form was not completed, it would not have been possible to 
carry out the mobilization in 720 (59\%) stay-days because patients failed to meet the clinical stability criteria; however, in the other 509 (41\%) stay-days, patients met the criteria and should have been mobilized. Our group recently developed and validated a tool that can help us to improve data collection through real-time random safety audits, which have proven effective in detecting and correcting errors of omission in real time, thereby improving adherence to guidelines [10]. Including the audit of the EM form in our routine real-time random safety audits will improve adherence to our EM protocol.

We found that EM started a median of 3 days after admission; this finding is similar to the time to mobilization reported by Basset et al.[25]. However, a recently published meta-analysis concluded that the time when patients are mobilized varies widely, ranging from the first to the eighth day after ICU admission[26]. It is important to note that some studies define the starting point of EM as the initiation of passive mobilizations[25] and others define it as the initiation of active mobilizations[27].

The incidence of adverse events (0.4\%), including no serious complications, in the three years analyzed in our study is even lower than those reported by 
Bourdin et al.[8] (3\%) and Bailey et al.[7] (<1\%), adding weight to their conclusions that EM is safe.

\section{Limitations}

Our study was conducted at a single center, so caution is warranted in extrapolating our results and conclusions to other ICU. Our EM protocol was designed following the recommendations of experts and published studies, adapting them to the resources available at our institution. The lack of guidelines to unify protocols in terms of the criteria for clinical stability, types of mobilization, and duration of treatment makes comparisons with other studies difficult.

Our study analyzed data stored in the CIS, and the reliability of some of these data depends on the staff's commitment to collecting and entering them during routine daily practice. In this regard, it is important to highlight that the ETL enabled us to determine that patients did not meet the safety criteria for mobilization in 509 of the 1229 stay-days for which no EM form was registered in the CIS (considered stay-days in which the protocol was not adhered to). It is possible that in some of these cases the staff decided not to perform EM without completing the form in the CIS, and failure to register the decision could account for up to $15.6 \%$ of the cases that would be classified as failure to 
adhere to the protocol.

\section{Conclusions}

Our study shows that it is possible to use data from the CIS to evaluate many aspects of an EM protocol during a long period of clinical practice. Although adherence to the protocol was high, patients were mobilized in only one-third of all stay-days. The information from this study points to ways we can improve EM in our ICU, including reviewing the sedation protocol, assessing the suitability of the criteria for clinical stability, and increasing the presence of physiotherapists, or if it is not possible to increase the presence of physiotherapists, trying to involve family members. Importantly, the entire study required no extra efforts in data collection thanks to staff registering information in the CIS in their daily routine. The lack of data for one-third of all the stay-days pointed out the need to implement mechanisms (e.g., real-time random safety audits) to audit routine data collection in our ICU.

\section{List of abbreviations}

APACHE II: Acute Physiology and Chronic Health Evaluation II; BI: barthel index; BMI: body mass index; CIS: clinical information system; EM: early mobilization; ETL: extract, transform, and load; ICU: intensive care units; LOS: 
length of stay; MV: mechanical ventilation; MV-T: mechanical ventilationtracheostomy; SOFA: Sequential Organ Failure Assessment; RASS: Richmond Agitation-Sedation Scale.

\section{Declarations}

\section{Ethics approval and consent to participate}

All patients or their legal representatives provided written informed consent, and our center's research ethics committee approved the study protocol.

\section{Consent for publication}

Not applicable

\section{Availability of data and materials}

The datasets used and/or analyzed during the current study are available from the corresponding author on reasonable request.

\section{Competing interests}

The authors declare that they have no competing interests.

\section{Funding}

This study was supported by grants from the Fondo de Investigación Sanitaria (Carlos III Institute of Health, Spain, FIS grants, project PI20/01674). Agència de Gestió d'Ajuts Universitaris i de Recerca 2017 SGR 00127. FEDER. 


\section{Authors' contributions}

All authors read and approved the final manuscript.

\section{Acknowledgements}

The authors would like to thank all the ICU physicians, nurses, nursing assistants, physiotherapist, rehabilitors and health care personnel for their commitment and contribution to the project.

\section{References}

1. Thomsen GE, Snow GL, Rodriguez L, Hopkins RO. Patients with respiratory failure increase ambulation after transfer to an intensive care unit where early activity is a priority. Crit Care Med. 2008;36:1119-24.

2. Chambers MA, Moylan JS, Reid MB. Physical inactivity and muscle weakness in the critically ill. Crit Care Med. 2009;37.

3. Stevens RD, Dowdy DW, Michaels RK, Mendez-Tellez PA, Pronovost PJ, Needham DM. Neuromuscular dysfunction acquired in critical illness: A systematic review. Intensive Care Med. 2007;33:1876-91.

4. Needham DM, Davidson J, Cohen H, Hopkins RO, Weinert C, Wunsch H, et al. Improving long-term outcomes after discharge from intensive care unit: Report from a stakeholders' conference. Crit Care Med. 2012;40:502-9.

5. Berney SC, Harrold M, Webb SA, Seppelt I, Patman S, Thomas PJ, et al. 
Intensive care unit mobility practices in Australia and New Zealand: A point prevalence study. Crit Care Resusc. 2013;15:260-5.

6. Nydahl P, Ruhl AP, Bartoszek G, Dubb R, Filipovic S, Flohr HJ, et al. Early mobilization of mechanically ventilated patients: A 1-day point-prevalence study in Germany. Crit Care Med. 2014;42:1178-86.

7. Bailey P, Thomsen GE, Spuhler VJ, Blair R, Jewkes J, Bezdjian L, et al. Early activity is feasible and safe in respiratory failure patients. Crit Care Med. 2007;35:139-45.

8. Bourdin G, Barbier J, Burle JF, Durante G, Passant S, Vincent B, et al. The feasibility of early physical activity in intensive care unit patients: A prospective observational one-center study. Respir Care. 2010;55:400-7.

9. Dale M. N. Mobilizing patients in the intensive care unit: Improving neuromuscular weakness and physical function. JAMA - J Am Med Assoc. 2008;300:1685-90.

10. Schweickert WD, Pohlman MC, Pohlman AS, Nigos C, Pawlik AJ, Esbrook CL, et al. Early physical and occupational therapy in mechanically ventilated, critically ill patients: a randomised controlled trial. Lancet [Internet]. Elsevier Ltd; 2009;373:1874-82.

11. Bodí M, Blanch LL, Maspons R. Los sistemas de información clínica: una oportunidad para medir valor, investigar e innovar a partir del mundo real. Med 
Intensiva. 2017;41:316-8.

12. Harrold ME, Salisbury LG, Webb SA, Allison GT. Early mobilisation in intensive care units in Australia and Scotland: A prospective, observational cohort study examining mobilisation practises and barriers. Critical Care; 2015;19:1-9.

13. Timenetsky KT, Neto AS, Assunção MSC, Taniguchi L, Eid RAC, Corrêa TD. Mobilization practices in the ICU: A nationwide 1-day point- prevalence study in Brazil. PLoS One. 2020;15:1-12.

14. Sirgo G, Esteban F, Gómez J, Moreno G, Rodríguez A, Blanch L, et al. Validation of the ICU-DaMa tool for automatically extracting variables for minimum dataset and quality indicators: The importance of data quality assessment. Int J Med Inform. 2018;112:166-72.

15. Hodgson CL, Stiller K, Needham DM, Tipping CJ, Harrold M, Baldwin CE, et al. Expert consensus and recommendations on safety criteria for active mobilization of mechanically ventilated critically ill adults. Crit Care. 2014;18:1-9.

16. Dubb R, Nydahl P, Hermes C, Schwabbauer N, Toonstra A, Parker AM, et al. Barriers and strategies for early mobilization of patients in intensive care units. Ann Am Thorac Soc. 2016;13:724-30.

17. Leditschke AI, Green M, Irvine J, Bissett B, Mitchell IA. What Are the 
Barriers to Mobilizing Intensive Care Patients? Cardiopulm Phys Ther J. 2012;23:26-9.

18. Fontela PC, Forgiarini LA, Friedman G. Clinical attitudes and perceived barriers to early mobilization of critically ill patients in adult intensive care units. Rev Bras Ter Intensiva. 2018;30:187-94.

19. Raurell-Torredà M, Arias-Rivera S, Martí JD, Frade-Mera MJ, ZaragozaGarcía I, Gallart E, et al. Degree of implementation of preventive strategies for post-ICU syndrome: Multi-centre, observational study in Spain. Enferm Intensiva. 2019;30:59-71.

20. Joseph I, McCauley R. Impact of Early Mobilization in the Intensive Care Unit on Psychological Issues. Crit Care Nurs Clin North Am. 2019;31:501-5.

21. Rukstele CD, Gagnon MM. Making strides in preventing ICU-acquired weakness: Involving family in early progressive mobility. Crit Care Nurs Q. 2013;36:141-7.

22. Devlin JW, Skrobik Y, Gélinas C, Needham DM, Slooter AJC, Pandharipande PP, et al. Clinical Practice Guidelines for the Prevention and Management of Pain, Agitation/Sedation, Delirium, Immobility, and Sleep Disruption in Adult Patients in the ICU. Crit Care Med. 2018.

23. Bodí M, Olona M, Martín MC, Alceaga R, Rodríguez JC, Corral E, et al. Feasibility and utility of the use of real time random safety audits in adult ICU 
patients: a multicentre study. Intensive Care Med. 2015;41:1089-98.

24. Bodí M, Oliva I, Martín MC, Gilavert MC, Muñoz C, Olona M, et al. Impact of random safety analyses on structure, process and outcome indicators: multicentre study. Ann Intensive Care. Springer Paris; 2017;7: 21

25. Bassett RD, Vollman KM, Brandwene L, Murray $\mathrm{T}$. Integrating a multidisciplinary mobility programme into intensive care practice (IMMPTP): A multicentre collaborative. Intensive Crit Care Nurs. 2012;28:88-97. 26. Tipping CJ, Harrold M, Holland A, Romero L, Nisbet T, Hodgson CL. The effects of active mobilisation and rehabilitation in ICU on mortality and function: a systematic review. Intensive Care Med. Springer Berlin Heidelberg; 2017;43:171-83.

27. Moss M, Nordon-Craft A, Malone D, Van Pelt D, Frankel SK, Warner ML, et al. A Randomized Trial of an Intensive Physical Therapy Program for Patients with Acute Respiratory Failure. Am J Respir Crit Care Med. 2016;193:1101-10. 


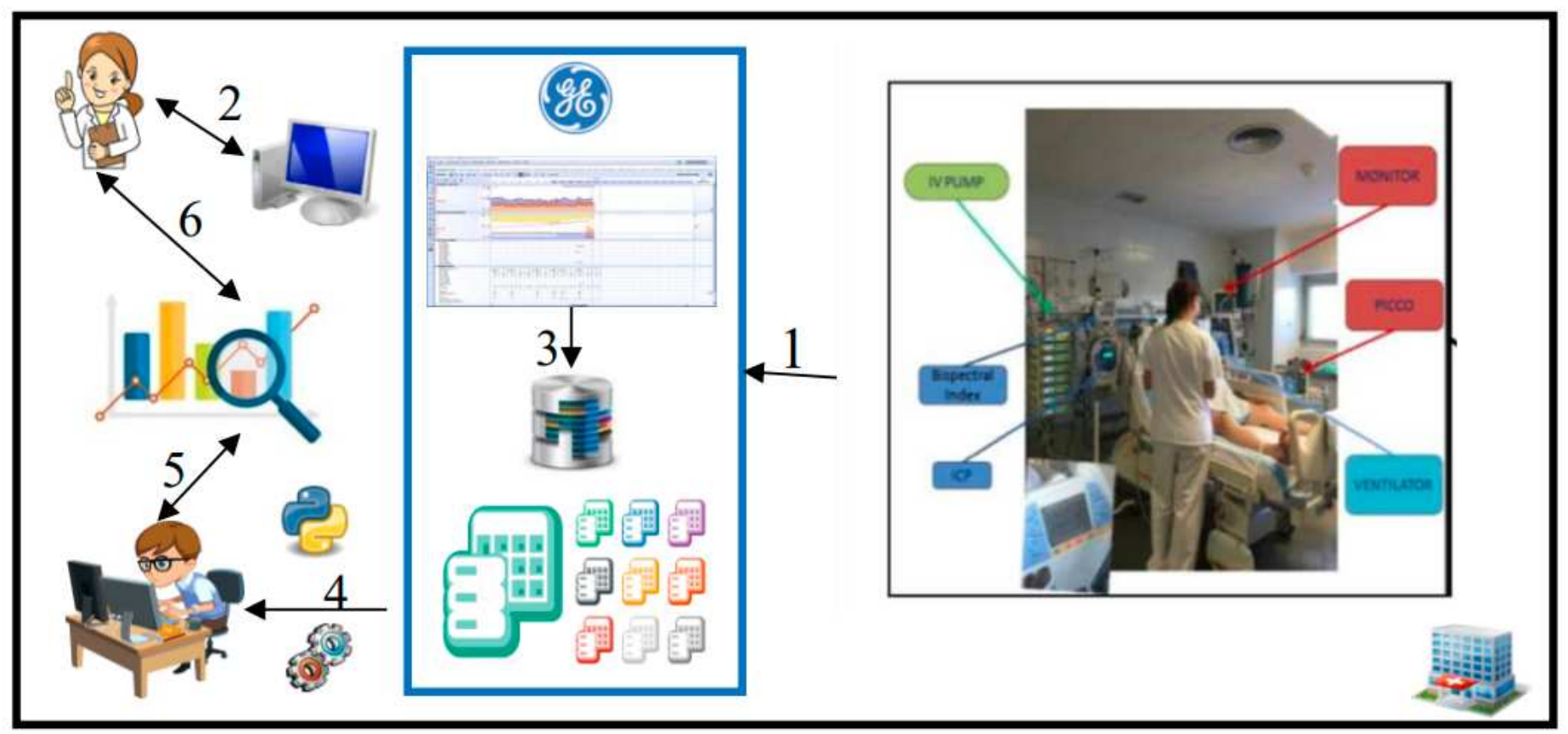

Figure 1

Flow of data in the ICU. The arrows show the flow of data from acquisition to display and analysis. Data from bedside devices are sent to the CIS (arrow 1), where they are stored in the database, organized in tables (arrow 3). Clinicians interact with the CIS, manually entering data and retrieving data through their own computers (arrow 2). Data scientists use ETL processes to integrate different types of data from different sources (arrow 4) and work together with clinicians to analyze the data statistically to interpret the data (arrows $5 \& 6$ ). 


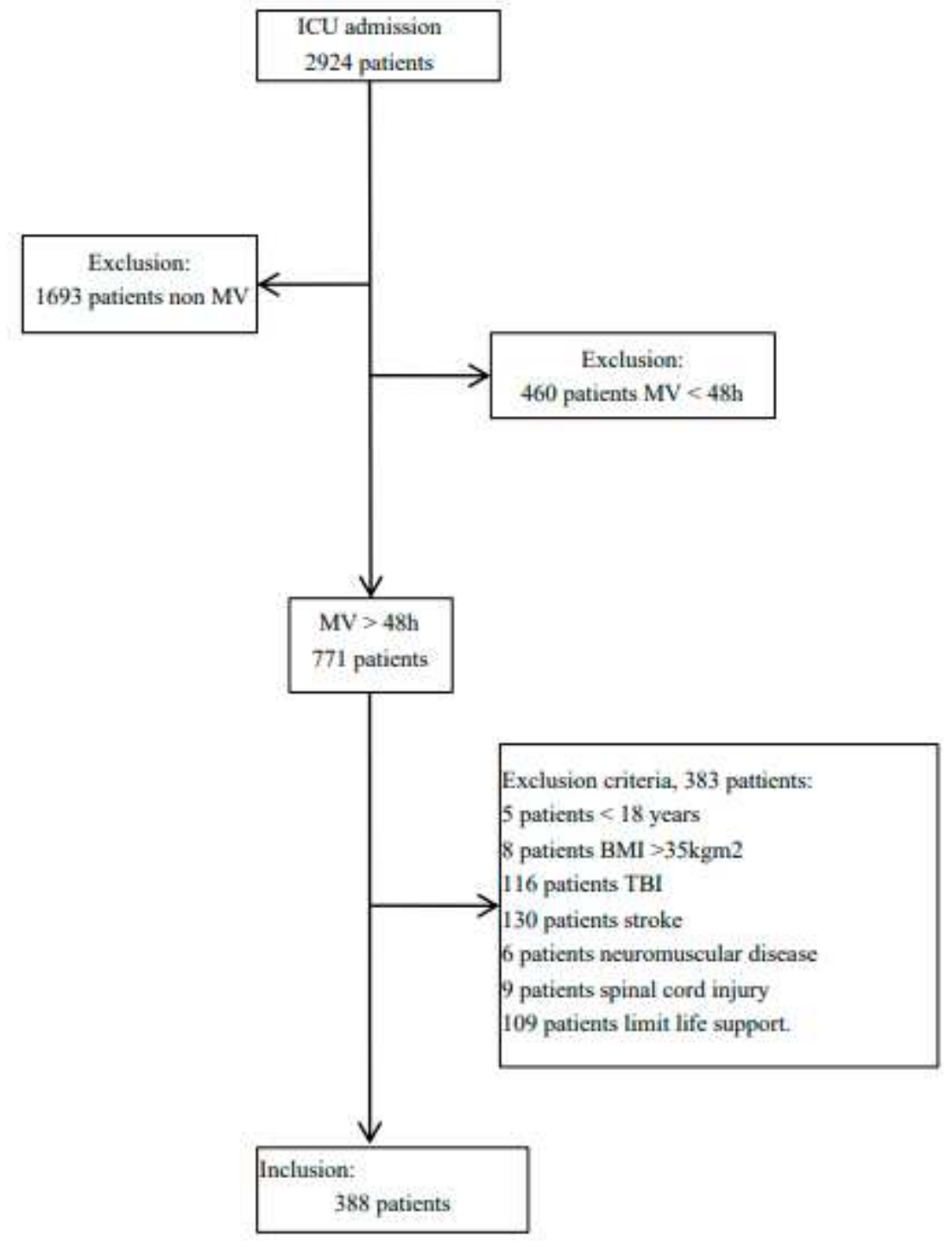

Figure 2

Flowchart study: number patients. ICU; intensive care unit, IMV; invasive mechanical ventilation, BMI; body mass index, TBl; trauma brain injury 


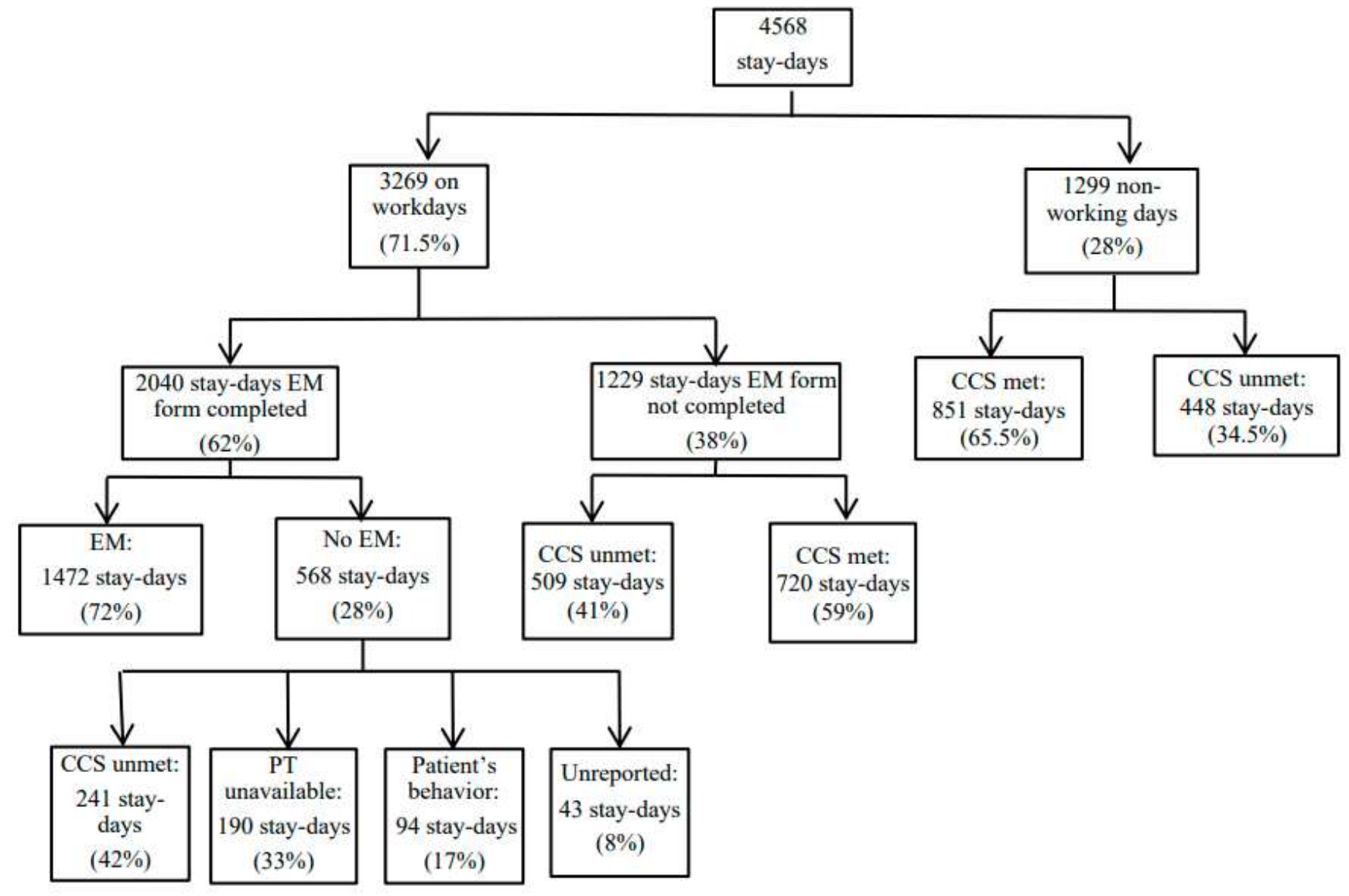

Figure 3

Flowchart showing reasons for not implementing the early mobilization protocol. EM: early mobilization; CCS: criteria for clinical stability; PT: physiotherapist

\section{Supplementary Files}

This is a list of supplementary files associated with this preprint. Click to download.

- Table1.pdf

- Additionalfiles.pdf 